\title{
Parsimonious DNA target-site recognition by Grh/CP2 transcription factors
}

$\underline{\text { Udo Heinemann }}^{1}$, Qianqian Ming ${ }^{1}$, Yvette Roske ${ }^{1}$, Anja Schuetz ${ }^{1}$, Kai M. Schmidt-Ott ${ }^{2}$

${ }^{1}$ Crystallography, Max Delbrück Center For Molecular Medicine, Berlin, Germany, ${ }^{2}$ Dept. of Nephrology, Charité Medical University, Berlin, Germany

E-mail: heinemann@mdc-berlin.de

Cell- and tissue-specific gene expression is controlled at the transcriptional and translational levels. Transcription factors bind to target sites on double-stranded DNA that are characterized by distinct nucleotide sequence motifs. Recently, we have studied two human transcription factors belonging to the Grainyhead/CCAAT-box-binding protein-2 (Grh/CP2) family which are shown to utilize an unusually parsimonious scheme of DNA target-site recognition (Ming et al., submitted).

Mammalian Grainyhead-like-1 (Grhl1), along with Grhl2, Grhl3 and the CP2 proteins, is grouped within a family of transcription factors for which no structural information was available until recently. These proteins are highly conserved in multicellular organisms as key regulators of epithelial differentiation, organ development and skin barrier formation, and several family members are linked to human disease $(1,2)$. Crystal structures of the DNA-binding domains (DBD) of human Grhl1 and Grhl2 were determined at $2.34-\AA$ and $2.50-\AA$ resolution and unexpectedly revealed a fold closely resembling the central tumor suppressor p53 in spite of very low sequence similarity. Subsequent structure analysis at $2.92-\AA$ resolution of the DNA target site-bound Grhl1 DBD showed 2:1 stoichiometry of protein chains to DNA duplex and a parsimonious mode of DNA sequence readout that relies on a small number of direct hydrogen bonds between the protein and DNA bases and additional contacts to the DNA backbone (Figure). Only two arginine residues per Grhl1 chain form direct hydrogen bonds to bases of the undistorted B-form DNA, which is in striking contrast to many other transcription factors forming multiple specific contacts to the DNA bases (e.g. ref. 3). This binding mode is fully supported by DNA-binding assays using mutated Grhl1 or Grhl2 variants and DNA target sites. The cancer-associated mutation arginine 427 to glutamine, in particular, leads to a complete abrogation of DNA target-sequence binding and reporter gene activation by Grhl1. The molecular mechanism of this loss of transcription factor function is revealed by the $2.35-\AA$ crystal structure of the Grhl1 R427Q DBD revealing a structural readjustment in the protein, triggered by the mutation, which destroys all direct contacts with DNA base pairs.

In summary, our studies establish the structural basis of DNA target-site recognition by Grh/CP2 transcription factors and the near complete loss of function associated with one single-site mutation.

1. Rifat, Y. et al. (2010). Dev. Biol. 345, 237-245.

2. Wang, S. \& Samakovlis, C. (2012). Curr. Topics Dev. Biol. 98, 35-44.

3. Schuetz, A. et al. (2011). Cell. Mol. Life Sci. 68, 3121-3131.

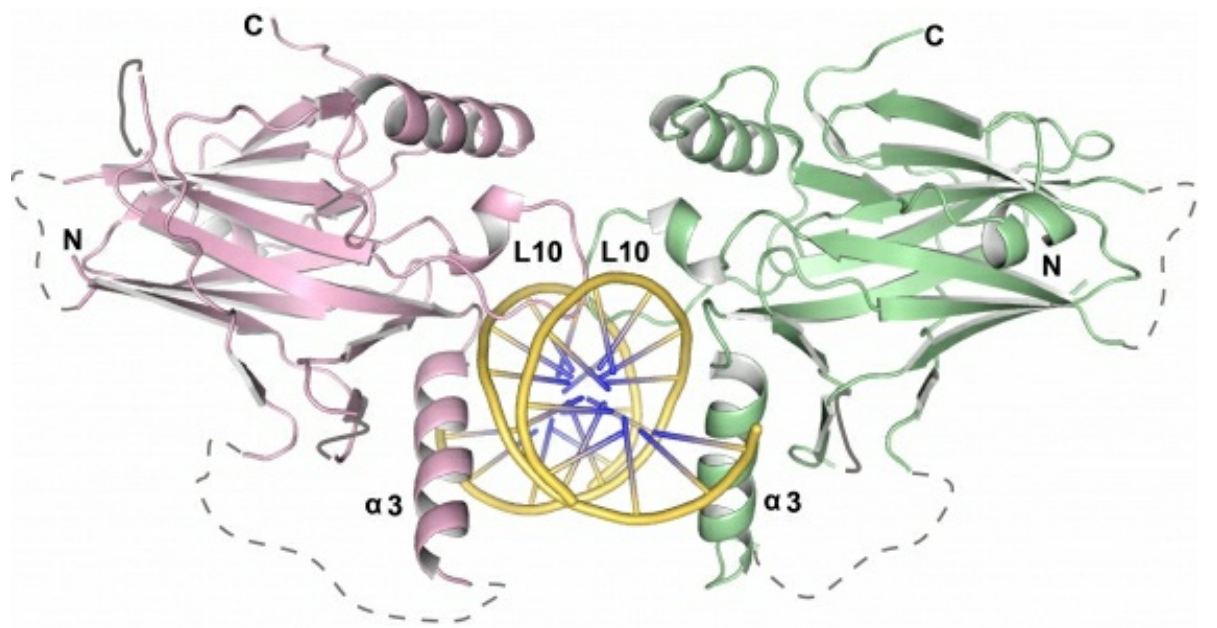

Keywords: protein-DNA recognition, transcription factors, Grainyhead/CP2 family 